\title{
The inter-annual distribution of cloudless days and nights in Abastumani: coupling with cosmic factors and climate change
}

\author{
G. G. Didebulidze, M. Todua \\ Abastumani Astrophysical Observatory, Ilia State University, \\ Kakutsa Cholokashvili ave. 3/5, 0162 Tbilisi. \\ e-mail.: didebulidze@iliauni.edu.ge
}

\begin{abstract}
Correspondin author: G. G. Didebulidze, KharadzeAbastumani Astrophysical Observatory at Ilia State University; K. Cholokashvili Ave 3/5; Tbilisi 0162; GEORGIA.

E-mail: didebulidze@iliauni.edu.ge
\end{abstract}

\begin{abstract}
We examined inter-annual variations and long-term trends of cloudless days (CD) and cloudless nights $(\mathrm{CN})$ in 1957-1993 from Abastumani (41.75 N, $42.82 \mathrm{E})$, at different geomagnetic conditions and corresponding galactic cosmic rays (GCRs) flux changes. It showed possible influence of cosmic factors on cloud covering processes and, thus, climate change. It was demonstrated that (1) the inter-annual distribution of monthly mean values of planetary geomagnetic index Ap (for low and moderate disturbances) at CDs can be described by harmonic function with semiannual (with sharp maxima in March and September) and annual (with maximum in August) periodicities; (2) the inter-annual distribution of Ap index for $\mathrm{CN}$ has an additional maximum in June, where the largest decrease of GCR flux is observed. This


phenomenon is expressed even stronger during Sudden Storm Commencement (SSC) events and strong geomagnetic disturbances ( $\mathrm{Ap} \geq 50$ ), when their relative numbers are the greatest and are accompanied by bigger reduction of GCRs flux; (3) the long-term trends of mean annual and mean seasonal values of Ap index and GCRs flux at $\mathrm{CD}$ and $\mathrm{CN}$ are estimated. It was detected that, for the latitudes of this region, long-term decreases (negative trends) of seasonal GCR flux are different at $\mathrm{CD}$ and $\mathrm{CN}$, which could affect the radiative balance at the Earth's surface and, as a result, contribute to the climate change.

\section{Keywords}

Cloud covering; Geomagnetic activity; Galactic cosmic rays; Climate change 


\section{Introduction}

Different variations of cloud covering during day- and night-time can have an effect on the radiative balance at the Earth's surface of a given region. This outcome is caused by different influence of clouds: during day-time they reflect the solar visual electromagnetic radiation, reducing its absorption by the Earth's surface, while during night-time they scatter and partly send back the Earth's infrared thermal radiation. This may have a significant impact on the Earth's radiative forcing and thus on the climate (Gray et al., 2010 and references therein). Therefore, it is important to consider inter-annual (seasonal) and long-term variations of cloudless days $(\mathrm{CD})$ and cloudless nights $(\mathrm{CN})$, which is the purpose of this study.

The clouds at various height levels may react on seasonal changes in atmospheric parameters in different ways, which could be caused by several factors, including cosmic ones (Marsh and Svensmark, 2000; Harrison and Carslaw, 2003; Tsonis et al., 2014). The presented study of interannual distributions and long-term changes of cloudless days and nights at various heliogeophysical conditions could contribute to the issue of possible impact of cosmic factors on cloud covering processes and its importance for climate change. For example, the long-term increase of the number of CD should enhance the absorption of solar EM radiation by the Earth's surface, while the decrease of the number of $\mathrm{CN}$ would reduce the loss of its thermal IR radiation.

To reveal possible input of cosmic factors in the inter-annual and long-term changes of CD and $\mathrm{CN}$, it is important to consider them for various geomagnetic disturbances, as well as solar activity and galactic cosmic rays (GCRs) flux variations. All these events, followed by some structural and possible dynamical changes of the atmosphere (Todua and Didebulidze, 2013), are coupled to each other and are important to reveal cosmic factors. Geomagnetic disturbances 
mainly follow active processes on the Sun and are expected to coincide with GCR flux decrease (Kudela and Brenkus, 2004). It is believed that GCRs are main source of ionization in the lower stratosphere and troposphere, below $30 \mathrm{~km}$ heights from the Earth's surface, and the reduction of its flux could decrease the amount of cloud condensation nuclei (Tinsley et al., 2006; Usoskin et al., 2009; Mishev et al., 2012). Cosmic rays determine the ionization rate and conductivities in the atmosphere and the ionosphere (below $90 \mathrm{~km}$ ) and thus the atmospheric electric fields. The latter influence thunderstorms, the Earth's global charge and global electric circuit between the ionosphere and the ground (Velinov, 2006). Thus, there could be a correlation between geomagnetic disturbances, as well as their consequent structural and dynamical changes and cloud covering processes in the lower atmosphere. The coupling between GCRs flux variations and geomagnetic activity also may be caused by changes in the GCR cut-off rigidity during these disturbances (Dorman, 2009).

This phenomenon, as well as the dependence of the frequency of occurrence of geomagnetic disturbances (with maxima at equinoxes) on the geometry of the interplanetary magneric field (IMF) and geomagnetic dipole (Russell and McPherron, 1973), can affect inter-annual variations of some atmospheric processes, as well as regional peculiarities of their development. GCRs flux changes following these geomagnetic disturbances can cause structural changes in the lower atmosphere. This can be reflected in the inter-annual variations of cloud covering, which are presented at some extent in the results of the current study.

To study the influence of these factors on cloud cover and consequent climate change, it is also important to consider long-term changes of $\mathrm{CD}$ and $\mathrm{CN}$ at various geomagnetic and solar activities, as well as at GCRs flux variations. These studies can develop the assumption that the centennial increase of solar activity (Mursula and Martini, 2006) is accompanied by decline in 
the GCR flux - the negative trend (Rouillard and Lockwood, 2007), that can cause the reduction of cloud covering and as a result can contribute to the global warming observed during last century.

In the present study we consider the inter-annual and long-term variations of cloudless days and cloudless nights in Abastumani for different levels of geomagnetic disturbances (described by planetary geomagnetic Ap index), corresponding day-night solar activity (described by solar radio flux $\mathrm{F}_{10.7}$ ) and GCRs flux changes. For the considered dataset, mean annual and mean seasonal Ap index and GCR flux trends for CD and CN will be estimated. Different changes of inter-annual/seasonal distributions of monthly mean Ap index and corresponding GCR flux will be determined, pointing at possible impact of cosmic factors on cloud cover process. Different seasonal negative trends of GCR flux for $\mathrm{CD}$ and $\mathrm{CN}$ also indicate cosmic factors influence on climate change in the considered region.

\section{Inter-annual variations of the planetary geomagnetic Ap index, GCRs and solar radio $F_{10.7}$ fluxes, at cloudless days and nights}

To reveal the influence of cosmic factors on cloud covering, in this section we consider the inter-annual distributions of the planetary geomagnetic Ap index, GCRs flux and solar radio flux F10.7 changes for CD and CN in Abastumani Astrophysical Observatory (AAO; 41.75N, 42.82E; $1600 \mathrm{~m}$ height a.s.1.), using the continuous data of $\mathrm{CD}$ and $\mathrm{CN}$, covering three 11-year solar cycles (1957-1993 years). During this period atmospheric studies by optical methods had been carried out almost continuously at AAO. Total ozone content had been observed during 
days, as well as nightglow observations at different wavelengths had been performed during lunarless nights. The observations required clear sky conditions during both day and night, which were estimated visually. Visually clear conditions in the whole field of view from the observatory are essential for middle and upper atmosphere monitoring by optical methods, which covers the area of almost 15-20 km in the troposphere (Megrelishvili, 1981; Fishkova, 1983). Within the referred period, the total number of CD was 4323 and that of $\mathrm{CN}$ was 1534 . Nightglow observations had been carried out only at lunarless cloudless nights. On Fig.1 the histogram of interannual distribution of the total monthly numbers of $\mathrm{CD}$ and $\mathrm{CN}$ in Abastumani (white and black columns, respectively) within the period 1957-1993 are presented. Total monthly numbers of CD vary from 227 to 531 , while those of $\mathrm{CN}$ from 78 to 199. The portion of visually cloudless days in the whole field of view is about $34 \%$ and that of nights at lunarless conditions is about $12 \%$ for the considered dataset. A large part of these data is available at www.woudc.org. This dataset allows us to investigate characteristics of the inter-annual (seasonal) distributions of $\mathrm{CD}$ and $\mathrm{CN}$ and examine them at different helio-geophysical conditions.

These data had been used to investigate different atmospheric parameters and their long-term variations by various authors (Megrelishvili 1981, Fishkova 1983, Givishvili et al. 1995; Gudadze et al. 2008, Didebulidze et al. 2011). In recent study (Todua and Didebulidze, 2013) the coupling of inter-annual/seasonal variations of some nightglow parameters with solar and geomagnetic activities were considered. It was demonstrated that they may be coupled with night-time cloudiness in the lower atmosphere.

Fig.1 demonstrates the different interannual/seasonal variations of $\mathrm{CD}$ and $\mathrm{CN}$. For example, the maximum number of CDs is in August, while that of CNs is in September. Since number of 
days in months slightly vary, we studied characteristics of the interannual variations of monthly mean values of various parameters (Ap, F10.7, GCRs), as well as relative numbers of CD and CN.

As a proxy of geomagnetic disturbance we use the planetary geomagnetic Ap index, which at some extent correlates with dynamical and structural changes of the mid-latitude atmosphereionoshere (Sinnhuber et al., 2012; Pancheva and Mukhtarov, 2011). We use this dataset to consider inter-annual distribution of the monthly mean planetary geomagnetic Ap index, GCRs flux changes and solar radio flux $\mathrm{F}_{10.7}$, at $\mathrm{CD}$ and $\mathrm{CN}$, for $\mathrm{Ap}<50$ (including quiet $0 \leq \mathrm{Ap} \leq 7$, unsettled $8 \leq \mathrm{Ap} \leq 15$, active $16 \leq \mathrm{Ap} \leq 29$ and minor storms $30 \leq \mathrm{Ap} \leq 49$, www.astrosurf.com/luxorion/qsl-perturbation5.htm).

Here, only $3.4 \%$ of day-nights are accompanied by strong geomagnetic disturbances ( $\mathrm{Ap} \geq 50$ ). For $96.6 \%$ data with $\mathrm{Ap}<50$ some regular inter-annual and long-term variations of the atmospheric parameters should be noticeable in their inter-annual distributions. The cases of sudden storm commencements (SSC) and strong geomagnetic disturbances with $\mathrm{Ap} \geq 50$ also will be considered, where atmospheric changes caused by solar-terrestrial coupling processes should be more expressed.

At first, we consider $\mathrm{CD}$ and $\mathrm{CN}$ with $\mathrm{Ap}<50$ and demonstrate the inter-annual variations of monthly mean values of the following quantities (Fig. 2): geomagnetic planetary Ap index (first panel from the top), solar radio $\mathrm{F}_{10.7}$ flux (second panel), GCRs fluxes (third and fourth panels). The values are calculated for all day-nights (dotted line), cloudless days (circles) and cloudless nights (dots) in Abastumani. For Ap and $\mathrm{F}_{10.7}$ the data are taken within 1957-1993 time interval. GCRs flux data had been taken from Tbilisi neutron monitor observations in 1964-1993 (third panel) and Rome neutron monitor (fourth panel) observations in 1957-1993 (www.izmiran.ru, 
ftp://cr0.izmiran.rssi.ru/COSRAY!/FTP_DDMM/Data/). For GCRs flux we take normalized values calculated as $X_{n}=(X-\bar{X}) / \bar{X}$, where $X$ is a mean daily GCR count, and $\bar{X}$ is the mean count averaged over the whole dataset with Ap<50. As GCR flux, along with the data of Tbilisi $\mathrm{NM}(\mathrm{Rc}=6.91 \mathrm{GeV})$, we use those of Rome NM with similar geomagnetic cut-off rigidity $(\mathrm{Rc}=6.32 \mathrm{GeV})$, which, unlike Tbilisi station, comprises the whole period of $\mathrm{CD}$ and $\mathrm{CN}$ data (1957-1993).

Fig. 2 shows that monthly mean Ap index has sharp maxima in March and September (for CD), while for all day-nights they appear smoother in February-March-April and SeptemberOctober periods. But for GCRs and $\mathrm{F}_{10.7}$ fluxes no significant changes in the periods of Ap maxima (or minima) are observed. The significant decrease of GCR flux is detected in June, where an additional maximum of $\mathrm{Ap}$ is observed for $\mathrm{CN}$, while for $\mathrm{CD}$ it has a minimum in this month. The sharp maxima of Ap index in March and September for CD, as well as the additional maximum in June for $\mathrm{CN}$, indicate possible influence of cosmic factors on cloud covering processes. We note, that Ap index, solar $\mathrm{F}_{10.7}$ radioflux and GCR flux depend considerably on solar phases. As a result, their fluctuations during several solar cycles are significant. Standard deviations (SD) for mean annual values are following: for Ap (<50) 9.5 (all day-nights), 9.5 (CD) and $10.0(\mathrm{CN})$; for $\mathrm{F}_{10.7} 56$ (all day-nights), $58(\mathrm{CD})$ and $57(\mathrm{CN})$; for GCR flux of Tbilisi NM 0.046 (all day-nights), 0.047 (CD) and $0.049(\mathrm{CN})$; for GCR flux of Rome NM 0.037 (all day-nights), $0.039(\mathrm{CD})$ and $0.040(\mathrm{CN})$. These SD values do not provide us with an additional information about the characteristics of interannual variations. For example, for $\mathrm{CN}$ in June, SDs for Tbilisi and Rome NM data, accompanying maximal Ap, are almost the same and do not differ significantly from those of other seasons. 
The sharp maxima of Ap index for CD in March and September indicates the existence of half-year (semiannual) periodicity (Katsavrias et al., 2012; Chowdhury et al., 2015) in this index, as well as it means the growth of magnetically disturbed CDs relative to all days and all CDs. Thus we can assume that, likewise, the full relative numbers of $\mathrm{CD}$ (or $\mathrm{CN}$ ) $R_{f}$, which we define as a ratio of number of $\mathrm{CD}$ (or $\mathrm{CN}$ ) in a month to all days in the same month, and the relative numbers of magnetically disturbed $\mathrm{CD}$ (or $\mathrm{CN}$ ) $R_{m}$, which we will denote MDCD (or MDCN) for short, and which we define as a ratio of number of MDCD (or MDCN) in a month to all $\mathrm{CD}$ (or $\mathrm{CN}$ ) in the same month, can be described by the equation (in analogy to Didebulidze and Todua, 2015):

$R_{f, m}(t, A p)=A_{6 f, m}(t) \cdot \cos \left[\frac{2 \pi}{T_{6}}(t-1)+\psi_{6}\right]+A_{12 f, m} \cdot \cos \left[\frac{2 \pi}{T_{12}}(t-1)+\psi_{12}\right]+c_{f, m}$,

where $t$ denotes months $(t=1,2, \ldots, 12)$ : January, February,... December; $A_{6 f, m}\left(A_{6 f}\right.$ or $\left.A_{6 m}\right)$ and $A_{12 f, m}\left(A_{12 f}\right.$ or $\left.A_{12 m}\right)$ are the amplitudes of $R_{f}$ or $R_{m}$ oscillations with semi-annual $\left(T_{6}=6\right)$ and annual $\left(T_{12}=12\right)$ periods, respectively; $\psi_{6}$ and $\psi_{12}$ are their phases in January $(\mathrm{t}=1)$. The full relative number of $\mathrm{CD}$ (or $\mathrm{CN}$ ) $R_{f}(t) \leq 1$ describes the mean yearly distribution of $\mathrm{CD}$ (or CN). Similarly, the relative numbers of MDCD (or MDCN) $R_{m}(t) \leq 1$ describe the inter-annual variations of MDCD (or MDCN) among $\mathrm{CD}$ (or $\mathrm{CN}$ ) during the year. For example, $R_{f}(t=8)=1$ means that in August all days (or all nights) were cloudless, $R_{m}(t=2, A p \geq 12)=0.25$ (or $\left.R_{m}^{c n}(t=8)=0.25\right)$ means that in February one out of four $\mathrm{CD}($ or $\mathrm{CN})$ in average was 
magnetically disturbed with $\mathrm{Ap} \geq 12 . c_{f}$ (or $c_{m}$ ) is a constant part for inter annual variations of $R_{f}\left(\right.$ or $\left.R_{m}\right)$

In Fig. 3 the inter-annual distributions of observed and theoretical data of the following values are demonstrated: top panel - full relative numbers $R_{f}(t$, all $A p)$ (circles - observed data, full line - fitted model curve), middle panel - full relative numbers of MDCD $R_{f}(t, A p \geq 12)$ (triangles - observed, dashed line - fitted model), $R_{f}(t, A p \geq 20)$ (square - observed, dotted line fitted model), and bottom panel - relative numbers of the MDCDs $R_{m}(t, A p \geq 12)$ and $R_{m}(t, A p \geq 20)$, respectively. Here the time-dependent amplitude $A_{6 f}(t)$ (or $\left.A_{6 m}(t)\right)$ for semiannual variations of $\mathrm{CD} R_{f}$ (or $R_{m}$ ), Eq. (1), with observed maxima in March (t=3) and September ( $\mathrm{t}=9)$, was described by the following equation:

$$
A_{6 f, m}(t)=a_{6 f, m} \cdot\left[\frac{1}{1+a_{6 f, m}^{\prime}(t-3)^{2}}+\frac{1}{1+a_{6 f, m}^{\prime}(t-9)^{2}}\right],
$$

where the parameters $a_{6 f}$ (or $\left.a_{6 m}\right)$ and $a_{6 f}^{\prime}\left(a_{6 m}^{\prime}\right)$ are estimated by fitting the observed values of $R_{f}$ (or $R_{m}$ ) to the empirical ones determined by Eqs. (1) and (2). The $A_{6 f}$ and $A_{6 m}$ amplitudes have peaks in March and September and fit quite well (Fig. 3) with the observed values of the $R_{f}$ and $R_{m}$ for the CD. For example, the coefficient of determination R-square of approximations by (1) and (2) are the following: for $R_{f}(t$, all $A p)-0.93$, for $R_{f}(t, A p \geq 12)$ 0.96 , for $R_{f}(t, A p \geq 20)-0.93$, in case of MDCD, for $R_{m}(t, A p \geq 12)$ and $R_{m}(t, A p \geq 20)-0.89$ and 0.91 , respectively. 
In the distribution of $\mathrm{CD}$ the annual variations with maxima in August $\left(\psi_{12}=\frac{5 \pi}{6}\right)$ are well established (Fig. 3, top panel), as well as semiannual variations $\left(\psi_{6}=-\frac{2 \pi}{3}\right)$, depending on Ap index with maxima in March and September (Fig. 3, middle panel). The semiannual variations are manifested even more in relative numbers of MDCD (Fig. 3, bottom panel) which also is given in the observed inter-annual distribution of monthly mean Ap (Fig. 2, top panel). We note that annual variations of monthly mean Ap for CD also are well described by (1) and (2), with Rsquare $=0.93$, though for all Ap this approximation is less good $(\mathrm{R}$-square $=0.85)$. This could point at the influence of cosmic factors on cloud cover. This impact also may differ for day- and nighttime.

Fig. 4 is similar to Fig. 3 but for $\mathrm{CN}$ : top panel - full relative numbers $R_{f}(t$, all $A p)$ of $\mathrm{CNs}$ (dots - observed data, full line - fitted model); middle panel - full relative numbers of MDCN $R_{f}(t, A p \geq 12)$ (triangles - observed, dashed line - fitted model) and $R_{f}(t, A p \geq 20)$ (square observed, dotted line - fitted model); bottom panel - relative numbers of the MDCNs to all CNs $R_{m}(t, A p \geq 12)$ and $R_{m}(t, A p \geq 20)$, respectively. Here $R_{f}$ and $R_{m}$, defined by (1), are not timedependent. Fig. 4 shows that for $\mathrm{CN}$, besides annual and semiannual variations described by Eq.(1), other periodicities also are possible.

For CN, $R_{f}$ of Eq.(1) describes at some extent the observed annual variations. For example, in case of all nights (Fig. 4, top panel) R-square $=0.74$, for $A p \geq 12$ and $A p \geq 20$ (Fig. 4, middle panel) R-square are equal to 0.78 and 0.64 , respectively. As for relative number of MDCN, $R_{m}$, where there are possibly other periodicities (Fig. 4, bottom panel), this description is not good enough (R-square $<0.5$ ). The maximal value of Ap in June (Fig. 2) is the result of the observed increase 
of $R_{m}$ during the growth of the level of geomagnetic disturbances. It is followed by even bigger decrease of the GCRs flux. This phenomenon is not described by harmonic functions. This increase of the relative numbers of MDCN in June, when, at the same time, GCR flux is decreased, may indicate the effect on cloud cover. We will consider it at SSC and strong geomagnetic disturbances.

In Fig. 5 the inter-annual distributions of the following quantities are presented: (top panels) relative monthly numbers of (right) Sudden Storm Commencement (SSC) and (left) strong geomagnetic disturbances with $\mathrm{Ap} \geq 50$, for 1957-93; (middle and bottom panels) corresponding monthly mean relative GCRs flux changes (see Fig. 2 for definition) observed by Tbilisi neutron monitor during 1964-1993 (middle panel) and Rome neutron monitor during 1957-1993 (bottom panel). Dashed lines represent the values for all day-night periods, circles with dash-dotted lines - those for CD, and dots with solid lines - for CN at Abastumani. We note that the Sudden Storm Commencements (SSC) also occur during strong and moderate geomagnetic disturbances and their number is greater than strong geomagnetic disturbances with Ap $\geq 50$. SSCs also accompany active processes on the Sun and thus GCR flux decrease is expectable. Strong geomagnetic disturbances $(\mathrm{Ap} \geq 50)$ are often accompanied by Forbush decrease (Kudela and Brenkus, 2004).

Fig. 5 shows that during SSC the inter-annual distribution of relative numbers of $\mathrm{CD}$ and $\mathrm{CN}$ are different, the maximum for $\mathrm{CN}$ being in June. GCR flux decrease for the same nights in June (and May) is about $8 \%$. Similar picture is for $\mathrm{Ap} \geq 50$ (right middle and bottom panels). Here the greatest number of geomagnetic disturbances for $\mathrm{CN}$ also occurs in June, accompanied by even larger drop of GCR flux of about 12\% (for Tbilisi and Rome). 
For other cosmic ray stations, the variations of GCR flux during a year for CD and CN are similar to those of Tbilisi and Rome neutron monitors. The greatest GCR flux decrease at CN in May-June is also visible for the stations with various cut-off rigidities $\left(\mathrm{R}_{\mathrm{c}}\right)$, namely Deep River $(\mathrm{Rc}=1.02 \mathrm{GeV})$, Moscow $(\mathrm{Rc}=2.46 \mathrm{GeV})$, Climax $(\mathrm{Rc}=3.03 \mathrm{GeV})$. For these stations in 1957-1993 period, the maximum decrease for $\mathrm{CN}$ in June at $\mathrm{SSC}$ is around $-(0.09 \div 0.12)$, and at $\mathrm{Ap} \geq 50$ is $-(0.15 \div 0.17)$. Such a reduction of GCR flux is typical for Forbush decrease, which often takes place at strong geomagnetic disturbances (Kudela and Brenkus, 2004). Thus, the drop of GCR flux for CN in June (Fig. 2) is even larger at strong geomagnetic disturbances (Fig. 5), which may indicate its influence on cloud cover.

From Figures 2-5 the different day- and night-time inter-annual (seasonal) variations of Ap index and GCR flux are seen, which can have an effect on the radiative balance at the Earth's surface in this region. This day- and night-time difference stimulates the study of long-term trends of Ap and GCRs flux, which will be considered in the next section.

\section{Long-term trends of the planetary geomagnetic Ap index and GCRs flux changes for cloudless days and nights}

Like solar activity, the number of geomagnetic disturbances and thus planetary geomagnetic Ap index, as well as GCRs flux, undergo variations with 11-year, secular and possibly other periods (Dorman 2009; Usoskin, 2013). The atmospheric parameters of different regions, experiencing seasonal changes, respond to solar and geomagnetic activities and their long-term variations (Fishkova, 1983; Megrelishvili, 1981) in different ways. As was demonstrated in previous section, the inter-annual distributions of Ap index and GCRs flux are different for CD and $\mathrm{CN}$ (Fig. 2). This can be explained by various distributions of relative numbers of CD and 
$\mathrm{CN}$ (Figs. 3 and 4). Different numbers of $\mathrm{CD}$ and $\mathrm{CN}$ in seasons, at various geomagnetic disturbances and GCR flux changes, should indicate their impact on radiative balance at the Earth's surface in this region. Thus their long-term variations should suggest their possible effect on climate change. Here we examine long-term variations and trends of Ap index and GCR flux for their mean annual and seasonal values. To calculate the trends for GCR flux changes we use again the data of the Rome neutron monitor of 1957-1993 period.

In Fig. 6 the long-term variations and linear trends of the mean annual planetary geomagnetic Ap (<50) index (upper panels) and corresponding GCRs flux changes (lower panels) for CD (circles and full line) and CN (dots and full line) during 1961-1993, for quiet and moderate geomagnetic disturbances $(\mathrm{Ap}<50)$, are plotted. The trends are demonstrated by straight lines: dashed lines for all day-nights, full ones for $\mathrm{CD}$ and $\mathrm{CN}$ (on the left and right plots, respectively). Both Ap index and GCRs flux vary significantly during solar minimum and maximum phases. Therefore, we estimated trends for 1961-1993 period which comprise three almost complete solar cycles. The Ap index trend for CD is $(0.051 \pm 0.053)$ year $^{-1}$ and for $\mathrm{CN}(0.053 \pm 0.094)$ year $^{-1}$, which are close to the one for all day-night - $(0.055 \pm 0.030)$ year $^{-1}$. The errors are given within 95\% confidence level. We note that these trends of mean annual values correspond to the mean seasonal daily ones, given below, which slightly differ from each other.

The GCRs flux trend values are negative: $(-0.0012 \pm 0.0023)$ year $^{-1}$ for $\mathrm{CD},(-0.0011 \pm 0.0022)$ year $^{-1}$ for $\mathrm{CN}$ and also are similar to all day-night case which is equal to $(-0.0012 \pm 0.0021)$ year $^{-1}$. The errors are given within $95 \%$ confidence level. These trends are estimated by mean yearly values of GCRs flux changes. The estimation by daily values gives the following trends: for all day-nights $\left(-3.2 \cdot 10^{-6} \pm 3 \cdot 10^{-7}\right)$ day $^{-1}$, for $\mathrm{CD}\left(-3.4 \cdot 10^{-6} \pm 6 \cdot 10^{-7}\right)$ day $^{-1}$, for $\mathrm{CN}\left(-2.9 \cdot 10^{-6} \pm 10^{-6}\right)$ day $^{-1}$. These values for mean yearly trends (365.25 days) are about the same as mentioned above, 
but significant. Similar negative trends are also observed for other GCR stations and may express the tendency of GCR flux decrease (Rouillard and Lockwood, 2007), accompanying the increase of geomagnetic disturbances observed during last century (Mursula and Martini, 2006; Martini et al., 2012). Unlike Ap and GCRs flux, the long-term trends of the solar radio flux (for $A p<50$ ) are smaller and are not demonstrated here. Fig. 6 demonstrates that the mean annual distributions of Ap $(\mathrm{Ap}<50)$ and GCR flux at cloudless days and nights differ slightly from those for all daynights. Thus, their long-term variations and trends are similar. We note that $\mathrm{Ap}<50$, which includes $96.4 \%$ of the whole data, decreases the fluctuations which also makes it possible to consider mean seasonal changes and their trends. For different interannual variations observed in $\mathrm{CD}$ and $\mathrm{CN}$ (Figs. 1-5), the seasonal trends of corresponding Ap index and GCR flux values are also important for revealing possible impact of cosmic factors on both cloud covering processes and climate change.

On Table 1 the long-term trends (c.i. 95\%) of mean seasonal Ap (Ap<50) index GCRs flux (Almaty, Rome, Tbilisi) during 1961-1993 for all day-nights, cloudless days and cloudless nights are presented. For seasons we took three-months intervals symmetrical to equinoxes and solstyces. The trends of data of the Rome and Almaty neutron monitors comprise 1961-1993 period, while those of Tbilisi station are taken for 1964-1993. Almaty $(\mathrm{Rc}=6.66 \mathrm{GeV})$ also has the geomagnetic cut-off rigidity similar to that of Tbilisi NM. As we can see, seasonal trend values are similar to those of Rome NM, which for some seasons are different from those obtained for Tbilisi NM data. Table 1 shows that seasonal changes of Ap index are relatively small in case of all day-nights, but they differ considerably in case of $\mathrm{CN}$. The biggest difference is for Summer season (May-June-July). For CN the Ap trend is negative and equals to (-2.8 $10^{-}$ $\left.{ }^{4} \pm 2.4 \cdot 10^{-4}\right)$ day $^{-1}$, while in other seasons it is positive. At the same time, GCRs flux decrease is 
maximal in this season. The long-term decrease of GCR flux, as we mentioned previously, could cause the change in cloud cover. From Table 1 it is seen that GCRs flux decrease varies in different seasons. Its trends for $\mathrm{CD}$ and $\mathrm{CN}$ also differ which may have an effect on cosmic factors impact on climate change in this region.

\section{Discussion}

To reveal possible influence of cosmic factors on cloud covering process, as well as climate change, we studied the inter-annual and long-term variations of the geomagnetic planetary Ap index, GCRs flux and Solar radio flux $\mathrm{F}_{10.7}$ for visually cloudless days and nights from Abastumani, using the data of continuous atmospheric observations during 1957-1993. Variations in absorption of solar electromagnetic radiation in the lower atmosphere at the Earth's surface during day- and night-time and influence of temperature and humidity changes should provide different conditions for condensation and cloud covering processes in the troposphere (Kristjánsson et al., 2008). The regions like Abastumani, where anthropogenic and natural dust effects has minor influence (Kokkalis et al. 2012), are exceptional for investigation of possible impact of cosmic factors on cloud formation processes. In this case, the conditions of a high altitude location may be important, since evaporation and fast temperature variations in the lower regions of cloud formation (<3.5 km a.s.1.), where GCR influence is more effective (Marsh and Svensmark, 2000; Calisto et al., 2011; Tsonis et al., 2014), are different from those of low altitudes. 
Investigation of the inter-annual and long-term variations of cloudless days and nights at different helio-geophysical conditions are important for revealing both the impact of cosmic factors on cloud covering processes and its influence on climate change. Clear days and nights affect the Earth's radiative balance during various seasons differently and their long-term changes can have an impact on warming or cooling tendencies in the region. The GCR flux decrease, resulting from its negative trend, should cause the reduction of cloud cover and could influence climate change. But for a given season, when there can be different effects on cloud covering processes, the seasonal values of GCR trends are important to evaluate long-term variations of radiative balance at the Earth's surface.

The maximal values of Ap index for CD in March and September (Fig. 2) are caused by increase of relative numbers of CD at geomagnetic disturbances for these periods of a year (Fig. 3). This behavior is well described by the periodic harmonic functions (Eqs. 1 and 2), dependent on Ap index, with semiannual (maxima in March and September) and annual (maximum in August) periodicities. This description indicates that the annual periodicity of the full relative numbers of $\mathrm{CD}$ can be due to maximal mean day-night temperature which is characteristic for the most parts of the Caucasus region and which is favorable for cloudless days. The dependence of the number of $\mathrm{CD}$ on semiannual variations of Ap index (Fig. 3), with the sharp maxima in March and September (Eq. 2) may indicate the impact of cosmic factor on cloud cover.

The consideration of the inter-annual distribution of monthly mean values of Ap index also indicates that the GCR flux changes could be the cosmic factor affecting cloud cover. In this case, the monthly mean values of Ap, besides September, increases in June as well, which is accompanied by the greatest decrease of the GCR flux during a year (Fig. 2). Though for the solar radio flux $\mathrm{F}_{10.7}$ no significant changes are observed. Also, the maxima of the numbers of 
SSC and strong geomagnetic disturbances $(\mathrm{Ap} \geq 50)$ are expected around spring and fall equinoxes (Russell and McPherron, 1973), but for CN they are the greatest in June and are accompanied by even greater decrease of GCR flux than it is for Ap<50 (Fig. 5). Since the length of a day in June is the longest in the Northen hemisphere, the absorption of visual solar electromagnetic radiation is the greatest, which provides longer time for water evaporation during this season. For high altitude regions like Abastumani, a large decrease of the temperature in the lower troposphere after sunset provides favorable conditions for condensation and thus for cloud formation. In this case the ionization rate enhancement in the atmosphere, due to increase in the GCR flux (Tinsley et al., 2006; Usoskin et al., 2009; Mishev et al., 2012) and thus growth of CCN density should increase the cloud cover. But decrease of GCR flux, which is expected at geomagnetic disturbances, can cause the increase of the number of $\mathrm{CN}$ in June (Figs 3, 4, 5). It is important to compare seasonal variations of cloudless days and nights of the regions with different seasonal variations of meteorological parameters and, as a result, different impact of GCR on atmospheric structure (Eroshenko et al., 2010) and cloud covering processes. This problem is to be considered in future.

The number of geomagnetic disturbances, as well as GCR flux changes, are connected to solar activity. So, their coupling with day- and night-time cloud covering processes can be reflected on the long-term variations of these values during $\mathrm{CD}$ and $\mathrm{CN}$. This also can be used to study the influence of cosmic factors on climate change. For the considered dataset, the mean annual Ap index trend is positive and is accompanied by the negative trend of the GCR flux, which are almost the same for all day-nights, as well as CD and CN (Table 1). GCR flux decrease should cause the reduction of the cloud condensation nuclei (Tinsley et al., 2006; Rawal et al., 2013) and, as a result, the cloud cover, which in turn should be reflected in the radiation balance at the 

2007). Cloud cover reduction must be followed by increase of CD and CN. To study their influence on radiative balance during a year, the seasonal variations are to be considered. For the considered dataset, the GCR flux is decreasing during all seasons (negative trend) and is the greatest for the summer season (May-June-July). In addition to $\mathrm{CD}$ and $\mathrm{CN}$, for the mid-latitude neutron monitors (Tbilisi, Almaty, Rome), the GCR flux decrease (negative trend) during daytime is greater than during night-time. This should result in increase of the CD and thus the increase of absorption of the solar EM radiation (mostly in visible band) by the Earth's surface. Relatively slow decrease of GCR flux in case of $\mathrm{CN}$ (smaller negative trend than that at CD) indicates possible decrease of the number of $\mathrm{CN}$ and consequently smaller loss of the thermal IR radiation of the Earth's surface, which could contribute to the climate change in the region. All this shows the importance of cosmic rays for the processes of solar - atmospheric relations.We also note GCR influence on the chemistry and electrical parameters in the atmosphere. They influence actively ozone production and loss processes. GCR can transmit their solar modulation to the ozonosphere, which significantly controls the thermal regime and dynamics (including the one of the cloud system) of the lower atmosphere, i.e. the weather and climate (Velinov, 2000; 2006).

Semi-annual variations of cloud covering processes, revealed with annual ones, which correlate with geomagnetic disturbances, stimulate the study of structural changes in the lower atmosphere for various helio-geophysical conditions, which can vary with height. Quick thermal processes during cloud formation can also have an effect on generation of atmospheric waves and be reflected in lower and upper atmospheric couplings, where the annual and semiannual variations 


\begin{abstract}
of upper atmospheric and ionospheric parameters are exhibited (Didebulidze et al., 2011; Didebulidze and Todua, 2015).
\end{abstract}

\title{
5. Conclusions
}

We examined inter-annual variations and long-term trends of geomagnetic Ap index, solar radio flux and GCRs flux at cloudless days (CD) and cloudless nights (CN) in 1957-1993 from Abastumani (41.75 N, 42.82 E). It was demonstrated that the inter-annual distribution of monthly mean values of planetary geomagnetic index Ap (for Ap<50) at CDs is described by harmonic functions with semiannual (with sharp maxima in March and September) and annual (with maxima in August) periodicities, which is caused by variations similar to those of relative numbers of magnetically disturbed $\mathrm{CN}$.

Inter-annual distribution of $\mathrm{Ap}$ index for $\mathrm{CN}$ has an additional maximum in June, where the largest decrease of GCR flux during a year (for Tbilisi neutron monitor and in May and June for Rome NM) is observed. This phenomenon is even more expressed during Sudden Storm Commencement (SSC) events and strong geomagnetic disturbances (Ap $\geq 50$ ), when their relative numbers are the greatest and are accompanied by bigger reduction of GCRs flux. We consider these observed phenomena as a possible manifestation of the influence of cosmic factors (GCRs flux variations) on cloud cover.

For a given dataset we estimated the long-term trends of mean annual and mean seasonal values of Ap index and GCRs flux at CD and CN. For GCRs flux we took the time period with three solar activity phases. It was detected that, for the latitudes of this region, the long-term decrease (negative trends) of mean seasonal GCRs flux changes are different at CD and CN, 
which could affect the radiative balance at the Earth's surface and, as a result, contribute to the climate change.

\section{Acknowledgment.}

This study is supported by Georgian Shota Rustaveli National Science Foundation grant No. $13 / 09$.

\section{References}

Calisto, M., Usoskin, I., Rozanov, E., Peter, T., 2011. Influence of Galactic Cosmic Rays on atmospheric composition and dynamics. Atmos. Chem. and Phys. 11, Issue 9, 4547 -4556.

Chowdhury, P., Choudhary, D.P., Gosain, S., Moon, Y.-J., 2015. Short-term periodicities in interplanetary, geomagnetic and solar phenomena during solar cycle 24, Astrophys. Space Sci. 356, 7-18.

Didebulidze, G.G., Lomidze, L.N., Gudadze, N.B., Pataraya, A.D., Todua, M., 2011. Long-term changes in the nightly behaviour of the oxygen red $630.0 \mathrm{~nm}$ line nightglow intensity and trends in the thermospheric meridional wind velocity. Int. J. Remote Sens., 32, 3093-3114.

Didebulidze, G.G., Todua, M., 2015. Investigation of presence of cosmic factors in the interannual distributions of cloudless days and nights in Abastumani. Sun and Geosph., 10(1), 5963.

Dorman, L., 2009. Cosmic rays in magnetospheres of the Earth and other planets. Astrophysics and Space Science Library, Volume 358. Springer Netherlands. 
Eroshenko E., Velinov, P.I.Y., Belov, A., Yanke, V., Pletnikov, E., Tassev, Y., Mishev, A., Mateev, L., 2010. Relationships between neutron fluxes and rain flows. J. Adv. Space Res., $46,637-641$.

Fishkova, L.M., 1983. The night airglow of the Earth midlatitude upper atmosphere. Metsniereba Press, Tbilisi (in Russian).

Givishvili, G.V., Leshchenko, L.N., Shmeleva, O.P., Ivanidze, T.G., 1995. Climatic trends of the mid-latitude upper atmosphere and ionosphere. J. Atm. Terr. Phys., 57, 871-874.

Gray, L.J., Beer, J., Geller, M., Haigh, J.D., Lockwood, M., Matthes, K., Cubasch, U., Fleitmann, D., Harrison, G., Hood, L., Luterbacher, J., Meehl, G. A., Shindell, D., van Geel, B., White W., 2010. Solar Influences on Climate. Rev. Geophys., 48, Issue 4.

Gudadze, N B., Didebulidze, G.G., Lomidze, L.N., Javakhishvili, G.SH., Marsagishvili, M.A., Todua M., 2008. Different long-term trends of the oxygen red $630.0 \mathrm{~nm}$ line nightglow intensity as the result of lowering the ionosphere F2 layer. Annales Geophys., 26, 2069-2080.

Harrison, R.G., Carslaw, K.S., 2003. Ion-aerosol-cloud processes in the lower atmosphere. Rev. Geophys., 41, Issue 3, 2.

Katsavrias, Ch., Preka-Papadema, P., Moussas, X., 2012. Wavelet analysis on Solar wind parameters and geomagnetic indices, Solar Phys. 280(2), 623-640.

Kirkby, J., 2007. Cosmic Rays and Climate. Surv. in Geoph., 28 (5-6), 333-375.

Kokkalis, P., Mamouri, R. E., Todua, M., Didebulidze, G.G., Papayannis, A., Amiridis, V., Basart, S., Pérez, C., Baldasano, J.M., 2012. Ground-, satellite- and simulation-based analysis of a strong dust event over Abastumani, Georgia, during May 2009. Int. J. Remote Sens., 33, Issue 16, 4886-4901. 
Kristjánsson, J.E., Stjern, C.W., Stordal, F., Fjaraa, A.M., Myhre, G., Jónasson, K., 2008.

Cosmic rays, cloud condensation nuclei and clouds - a reassessment using MODIS data. Atmos. Chem. Phys., 8, Issue 24, 7373-7387.

Kudela, K., Brenkus, R., 2004. Cosmic ray decreases and geomagnetic activity: list of events 1982-2002. J. Atmos. Sol. Ter. Phys., 66, 1121-1126.

Marsh, N.D., Svensmark, H., 2000. Low cloud properties influenced by cosmic rays. Phys. Rev. Lett., 85, 5004-5007.

Martini, D., Linthe, H.-J., Pandey, V. S., Lee, D.-H., 2012. On the centennial trend estimates of geomagnetic activity indices. J. Geophys. Res., 117, A06211.

Megrelishvili, T.G., 1981. Regularities of the variations of the scattered light and emission of the Earth twilight atmosphere. Metsniereba Press, Tbilisi (in Russian).

Mishev, A., Velinov, P.I.Y., Mateev, L., Tassev, Y., 2012. Ionization effect of nuclei with solar and galactic origin in the Earth atmosphere during GLE 69 on 20 January 2005. J. Atmos. Solar-Terr. Phys., 89, 1-7.

Mursula, K., Martini, D., 2006. Centennial increase in geomagnetic activity: Revised estimate based on the IHV index, J. Geophys. Res. 111, A08209.

Pancheva, D., Mukhtarov, P., 2011. Stratospheric warmings: The atmosphere-ionosphere coupling paradigm. J. Atmos. Sol. Terr. Phys., 73 (13), 1697-1702.

Rawal, A.,Tripathi, S.N., Michael, M.,Srivastava, A. K., Harrison, R.G., 2013. Quantifying the importance of galactic cosmic rays in cloud microphysical processes. J. Atmos. Sol. Terr. Phys., 102, 243-251.

Rouillard, A.P., Lockwood, M., 2007. Centennial changes in solar activity and the response of galactic cosmic rays. Adv. Space Res., 40, 1078-1086. 
Russell, C.T., McPherron, R.L., 1973. Semiannual Variation of Geomagnetic Activity. J. Geophys. Res., 78, Issue 1, 92-108.

Sinnhuber, M., Nieder, H., Wieters, N., 2012. Energetic Particle Precipitation and the Chemistry of the Mesosphere/Lower Thermosphere. Surv. Geophys. 33 (6), 1281 -1334.

Svensmark, H., Friis-Christensen, E., 1997. Variation of cosmic ray flux and global cloud coverage - a missing lin in solar-climate relationships. J. Atmos. Sol. Terr. Phys., 59, 12251232.

Tinsley, B.A., Zhou, L., Plemmons, A., 2006. Changes in scavenging of particles by droplets due to weak electrification in clouds. Atmos. Res., 79, 266-295.

Todua, M., Didebulidze, G.G., 2013. Cosmic factors influence on the inter-annual variations of the green $557.7 \mathrm{Nm}$ line and red $630.0 \mathrm{Nm}$ line nightglow intensities and their possible coupling with cloud covering at Abastumani $\left(41.75^{\circ} \mathrm{N}, 42.82^{\circ} \mathrm{E}\right)$. Acta Geophys., 62, Issue 2, 381-399.

Tsonis, A.A., Deyle, E.R., May, R.M., Sugihara, G., Swanson, K., Verbeten, J.D., Wang, G., 2014. Dynamical evidence for causality between galactic cosmic rays and interannual variation in global temperature. Procedings of National Academy of Sciences of USA, 112, 11.

Usoskin, I., 2013. A history of solar activity over millennia. Living Rev. Sol. Phys., vol. 10, no. 1.

Usoskin, I., Desorgher, L., Velinov, P.I.Y., Storini, M., Flueckiger, E., Buetikofer, R., Kovalstov, G.A., 2009. Ionization of the Earth's atmosphere by solar and galactic cosmic rays. Acta Geophys. 57, 88-101. 
Velinov, P.I.Y., 2000. Cosmic ray trigger effect in the galactic-solar-terrestrial physics, C. R. Acad. Bulg. Sci., 53(2), 37 - 40.

Velinov P.I.Y., 2006. Advancing our understanding of the cosmic ray processes that govern the solar influence on Earth and planets. Sun and Geosph. 1, 1, 5-7. 
Table 1. Long-term trends (c.i. 95\%) of mean seasonal Ap (Ap<50) index GCRs flux (Almaty, Rome, Tbilisi) during 1961-1993 for all day-nights, cloudless days and cloudless nights

\begin{tabular}{|c|c|c|c|c|c|}
\hline \multirow{2}{*}{$\begin{array}{c}\text { Condition } \\
\end{array}$} & Season & \multirow{2}{*}{ Ap trend, day } & \multicolumn{3}{|c|}{ GCRs trends, day } \\
\cline { 3 - 6 } & & & Almaty & Rome & Tbilisi \\
\hline \multirow{3}{*}{$\begin{array}{c}\text { All day- } \\
\text { nights }\end{array}$} & Feb-Mar-Apr & $2.5 \cdot 10^{-4} \pm 9.2 \cdot 10^{-5}$ & $-2.7 \cdot 10^{-6} \pm 8.2 \cdot 10^{-7}$ & $-3.6 \cdot 10^{-6} \pm 5.5 \cdot 10^{-7}$ & $-3.0 \cdot 10^{-6} \pm 5.5 \cdot 10^{-7}$ \\
\cline { 2 - 6 } & May-Jun-Jul & $2.4 \cdot 10^{-5} \pm 7.7 \cdot 10^{-5}$ & $-4.1 \cdot 10^{-6} \pm 8.2 \cdot 10^{-7}$ & $-3.6 \cdot 10^{-6} \pm 5.5 \cdot 10^{-7}$ & $-4.7 \cdot 10^{-6} \pm 5.5 \cdot 10^{-7}$ \\
\cline { 2 - 6 } & Aug-Sep-Oct & $1.5 \cdot 10^{-4} \pm 8.7 \cdot 10^{-5}$ & $-3.0 \cdot 10^{-6} \pm 8.2 \cdot 10^{-7}$ & $-3.0 \cdot 10^{-6} \pm 5.5 \cdot 10^{-7}$ & $-1.4 \cdot 10^{-6} \pm 8.2 \cdot 10^{-7}$ \\
\cline { 2 - 6 } & Nov-Dec-Jan & $1.9 \cdot 10^{-4} \pm 7.9 \cdot 10^{-5}$ & $-3.3 \cdot 10^{-6} \pm 5.5 \cdot 10^{-7}$ & $-3.3 \cdot 10^{-6} \pm 5.5 \cdot 10^{-7}$ & $-5.5 \cdot 10^{-6} \pm 5.5 \cdot 10^{-7}$ \\
\hline & & & & & \\
\hline \multirow{3}{*}{$\begin{array}{c}\text { Cloudless } \\
\text { days }\end{array}$} & Feb-Mar-Apr & $3.7 \cdot 10^{-4} \pm 1.9 \cdot 10^{-4}$ & $-3.0 \cdot 10^{-6} \pm 1.6 \cdot 10^{-6}$ & $-3.3 \cdot 10^{-6} \pm 1.1 \cdot 10^{-6}$ & $-4.9 \cdot 10^{-6} \pm 1.1 \cdot 10^{-6}$ \\
\cline { 2 - 6 } & May-Jun-Jul & $2.3 \cdot 10^{-5} \pm 1.2 \cdot 10^{-4}$ & $-4.9 \cdot 10^{-6} \pm 1.4 \cdot 10^{-6}$ & $-4.4 \cdot 10^{-6} \pm 1.4 \cdot 10^{-6}$ & $-5.5 \cdot 10^{-6} \pm 1.1 \cdot 10^{-6}$ \\
\cline { 2 - 6 } & Aug-Sep-Oct & $1.6 \cdot 10^{-4} \pm 1.3 \cdot 10^{-4}$ & $-3.3 \cdot 10^{-6} \pm 1.1 \cdot 10^{-6}$ & $-3.3 \cdot 10^{-6} \pm 1.1 \cdot 10^{-6}$ & $-2.7 \cdot 10^{-6} \pm 1.1 \cdot 10^{-6}$ \\
\cline { 2 - 6 } & Nov-Dec-Jan & $9.3 \cdot 10^{-5} \pm 1.8 \cdot 10^{-4}$ & $-3.6 \cdot 10^{-6} \pm 1.4 \cdot 10^{-6}$ & $-3.8 \cdot 10^{-6} \pm 1.4 \cdot 10^{-6}$ & $-2.5 \cdot 10^{-6} \pm 1.1 \cdot 10^{-6}$ \\
\hline \multirow{3}{*}{$\begin{array}{c}\text { Cloudless } \\
\text { nights }\end{array}$} & Feb-Mar-Apr & $4.9 \cdot 10^{-4} \pm 3.5 \cdot 10^{-4}$ & $-1.9 \cdot 10^{-6} \pm 2.2 \cdot 10^{-6}$ & $-1.9 \cdot 10^{-6} \pm 1.9 \cdot 10^{-6}$ & $2.7 \cdot 10^{-7} \pm 1.6 \cdot 10^{-6}$ \\
\cline { 2 - 6 } & May-Jun-Jul & $-2.8 \cdot 10^{-4} \pm 2.4 \cdot 10^{-4}$ & $-4.1 \cdot 10^{-6} \pm 3.0 \cdot 10^{-6}$ & $-3.3 \cdot 10^{-6} \pm 2.7 \cdot 10^{-6}$ & $-4.7 \cdot 10^{-6} \pm 1.9 \cdot 10^{-6}$ \\
\cline { 2 - 6 } & Aug-Sep-Oct & $2.5 \cdot 10^{-4} \pm 2.1 \cdot 10^{-4}$ & $-1.6 \cdot 10^{-6} \pm 1.6 \cdot 10^{-6}$ & $-1.9 \cdot 10^{-6} \pm 1.6 \cdot 10^{-6}$ & $-5.5 \cdot 10^{-7} \pm 2.5 \cdot 10^{-6}$ \\
\cline { 2 - 6 } & Nov-Dec-Jan & $2.0 \cdot 10^{-4} \pm 3.0 \cdot 10^{-4}$ & $-3.6 \cdot 10^{-6} \pm 1.6 \cdot 10^{-6}$ & $-3.0 \cdot 10^{-6} \pm 1.6 \cdot 10^{-6}$ & $-2.7 \cdot 10^{-7} \pm 1.6 \cdot 10^{-6}$ \\
\hline
\end{tabular}




\section{Figure caption}

\section{Inter-annual distributions of cloudless days and cloudless nights}

Fig. 1. The histogram of interannual distribution of total monthly numbers of cloudless days (white columns) and cloudless nights (black columns) in Abastumani within the period 19571993.

\section{Inter-annual distributions of Ap index, solar radio flux $\mathrm{F}_{\mathbf{1 0 . 7}}, \mathrm{GCRs}$ flux and}

Fig. 2. The inter-annual distributions of monthly mean values of the following quantities (with Ap <50): (first panel) Ap index; (second panel) solar radio flux $F_{10.7}$, the normalized GCR fluxes observed by (third panel) Tbilisi neutron monitor during 1964-1993 and (fourth panel) Rome neutron monitor during 1957-1993. Normalized GCR values are calculated by $(X-\bar{X}) / \bar{X}$, where $X$ are GCR counts and $\bar{X}$ is the mean of all data for Ap $<50$ observed by Tbilisi and Rome neutron monitors during 1964-1993 and 1957-1993, respectively. Dotted lines are for all day-nights, circles with dashed lines - for cloudless days, and dots with solid lines - for cloudless nights at Abastumani in 1957-1993.

\section{Empirical description of inter-annual distribution of cloudless days}

Fig. 3. The inter-annual distributions of observed and fitted data of the following values: top panel - full relative numbers $R_{f}(t$, all $A p)$ of CDs (circles - observed data, full line - a theoretical approximation); middle panel - full relative numbers of $\operatorname{MDCD} R_{f}(t, A p \geq 12$ ) (triangles observed, dashed line - fitted model) and $R_{f}(t, A p \geq 20)$ (square - observed, dotted line - fitted model); bottom panel - relative numbers of the same MDCDs to all CDs $R_{m}(t, A p \geq 12)$ and $R_{m}(t, A p \geq 20)$, respectively. 


\section{Empirical description of inter-annual distribution of cloudless nights}

Fig. 4. The inter-annual distributions of observed and fitted data of the following values: top panel - full relative numbers $R_{f}(t, a l l A p)$ of CNs (dots - observed data, full line - a fitted model); middle panel - full relative numbers of $\operatorname{MDCN} R_{f}(t, A p \geq 12)$ (triangles - observed, dashed line - fitted model) and $R_{f}(t, A p \geq 20)$ (square - observed, dotted line - fitted model); bottom panel - relative numbers of the same MDCNs to all $\mathrm{CNs} R_{m}(t, A p \geq 12)$ and $R_{m}(t, A p \geq 20)$, respectively.

\section{Inter-annual distributions of the numbers of strong geomagnetic disturbances}

Fig. 5. The inter-annual distributions of the following quantities are presented: (top panels) relative monthly numbers of (right) Sudden Storm Commencement (SSC) and (left) strong geomagnetic disturbances with $\mathrm{Ap} \geq 50$, for 1957-93; corresponding monthly mean GCRs flux changes (see Figure 2 for definition) observed by (middle panels) Tbilisi neutron monitor during 1964-1993 and (bottom panels) Rome neutron monitor during 1957-1993. Dashed lines represent the values for all day-night periods, circles with dash-dotted lines - those for cloudless days, and dots with solid lines - for cloudless nights at Abastumani.

\section{Long-term changes of Ap index and galactic cosmic rays (GCRs) flux}

Fig. 6. Long-term variations and linear trends of the mean annual planetary geomagnetic Ap index (upper panels) and GCRs flux changes (lower panels) for cloudless days (circles and full line) and cloudless nights (dots and full line), at $\mathrm{Ap}<50$. Trends are demonstrated by straight lines: dashed line for all day-nights, full one for CD and CN, accordingly. 


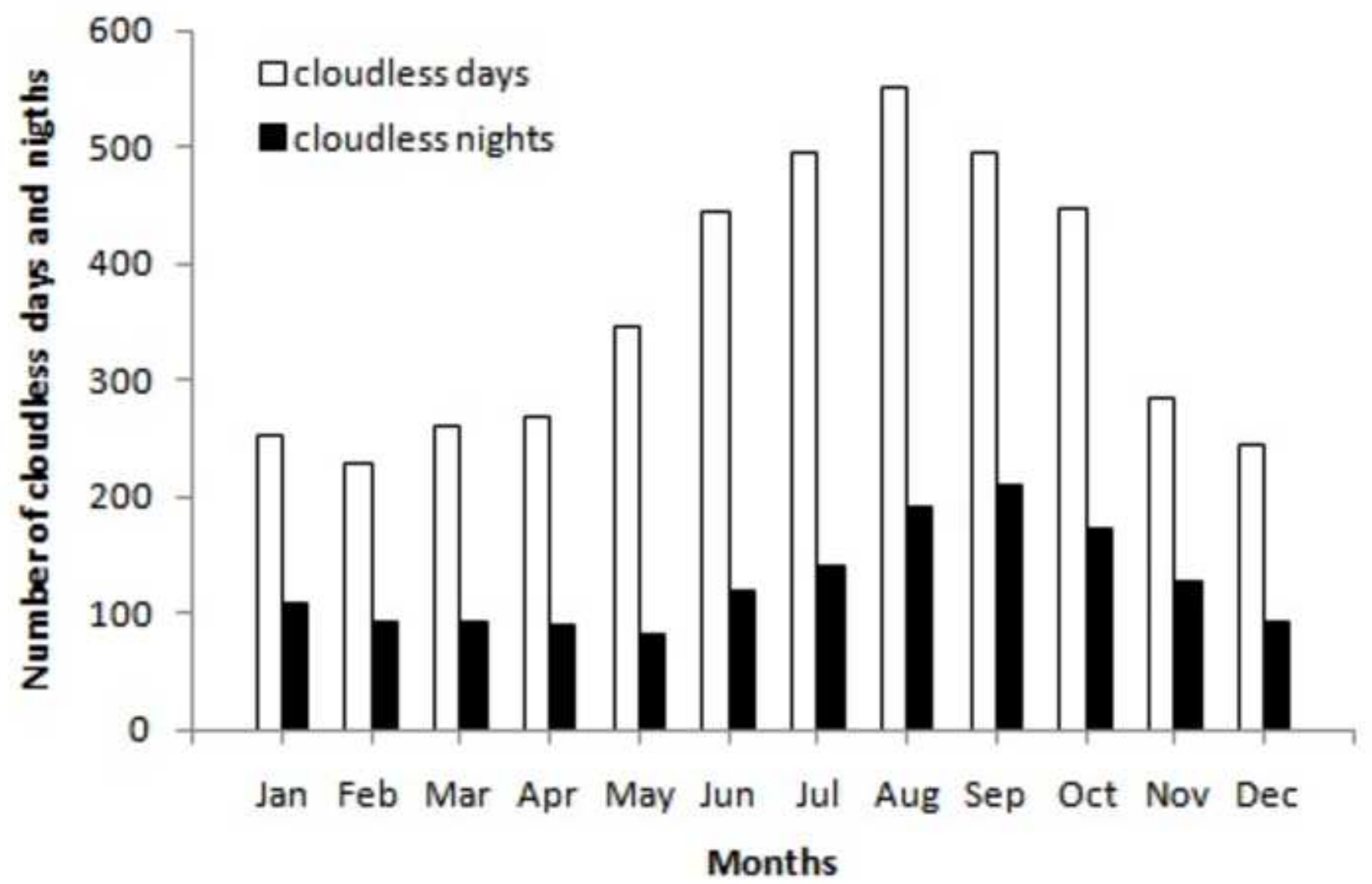



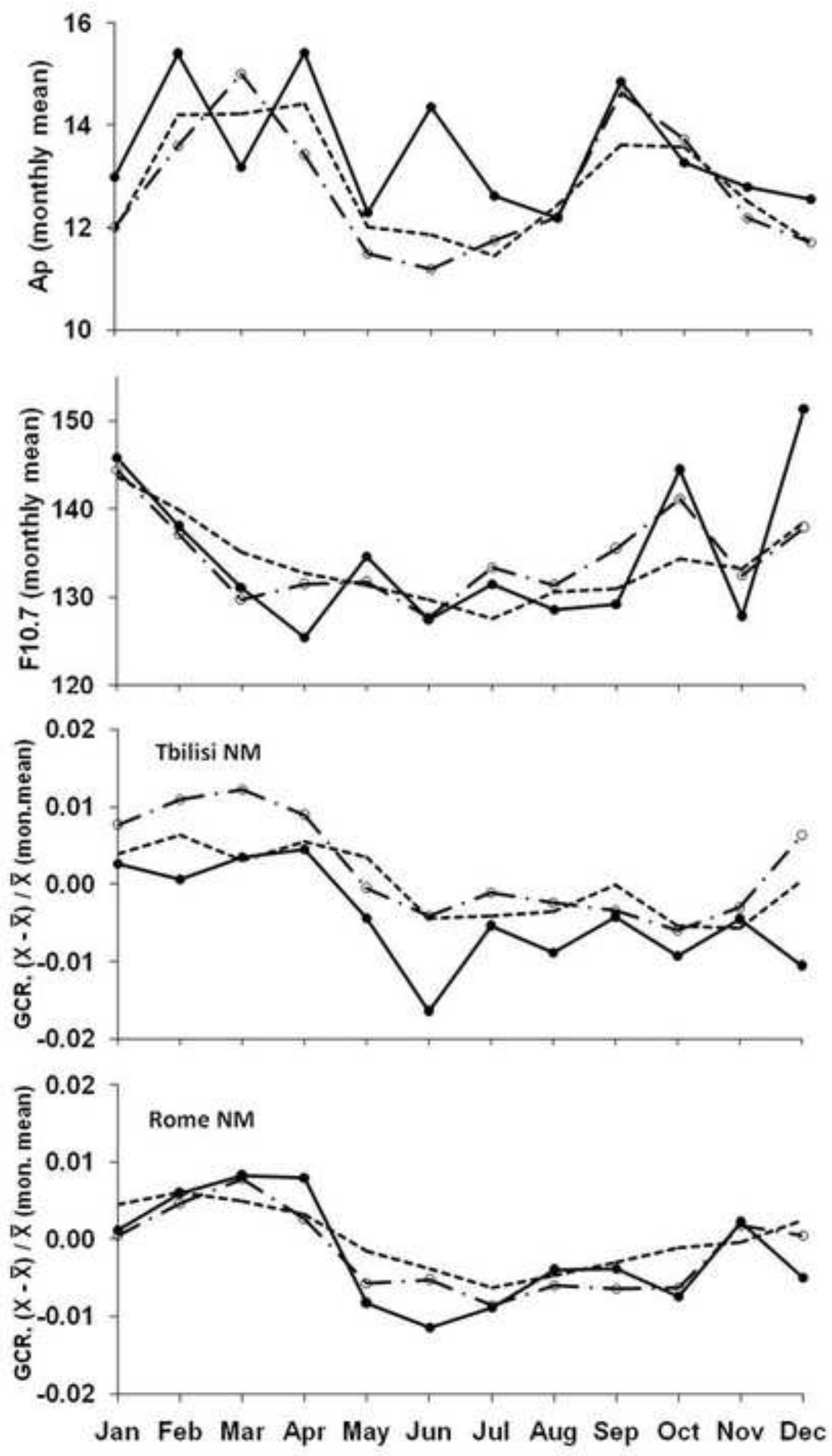
Figure 3
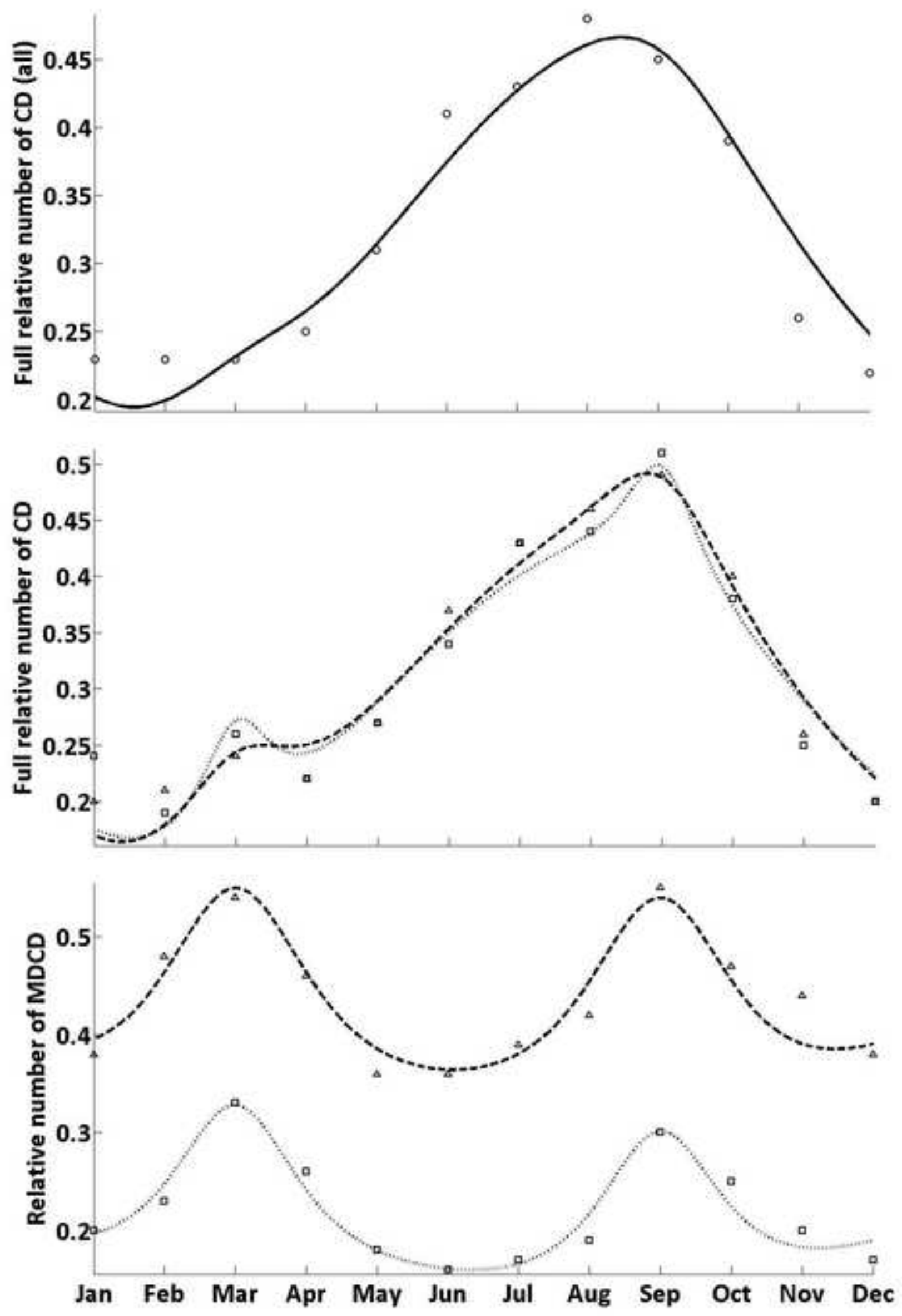

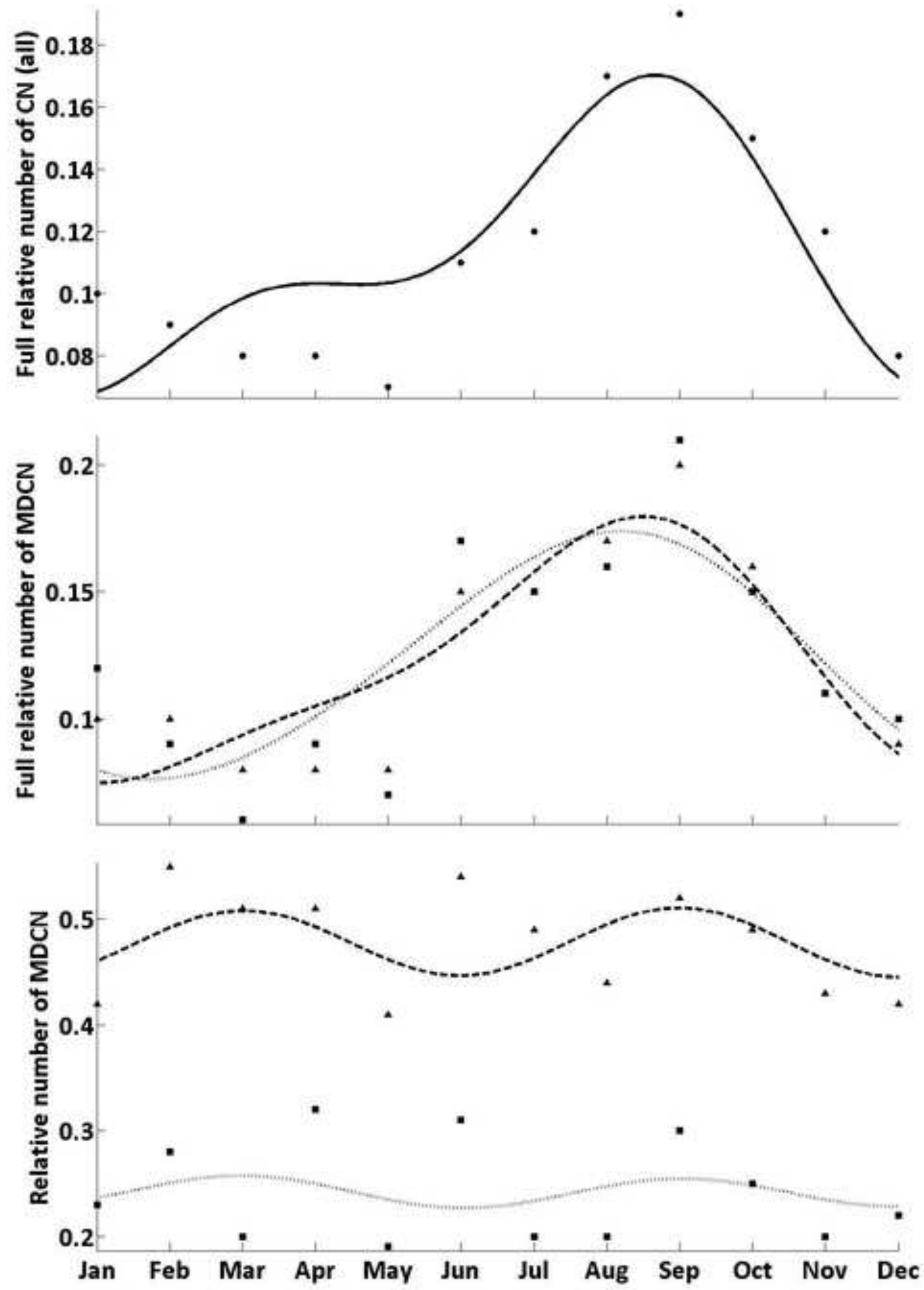

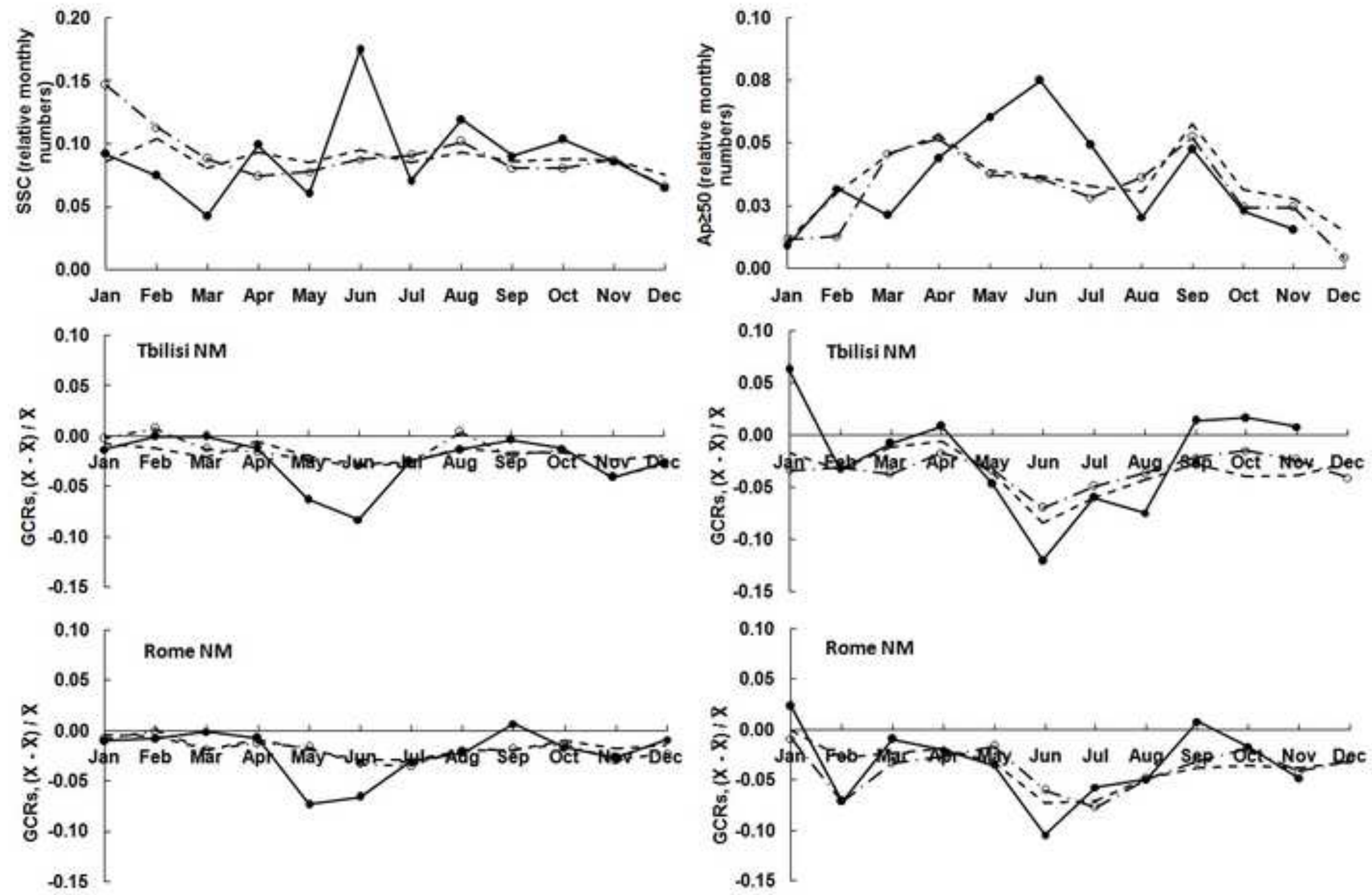

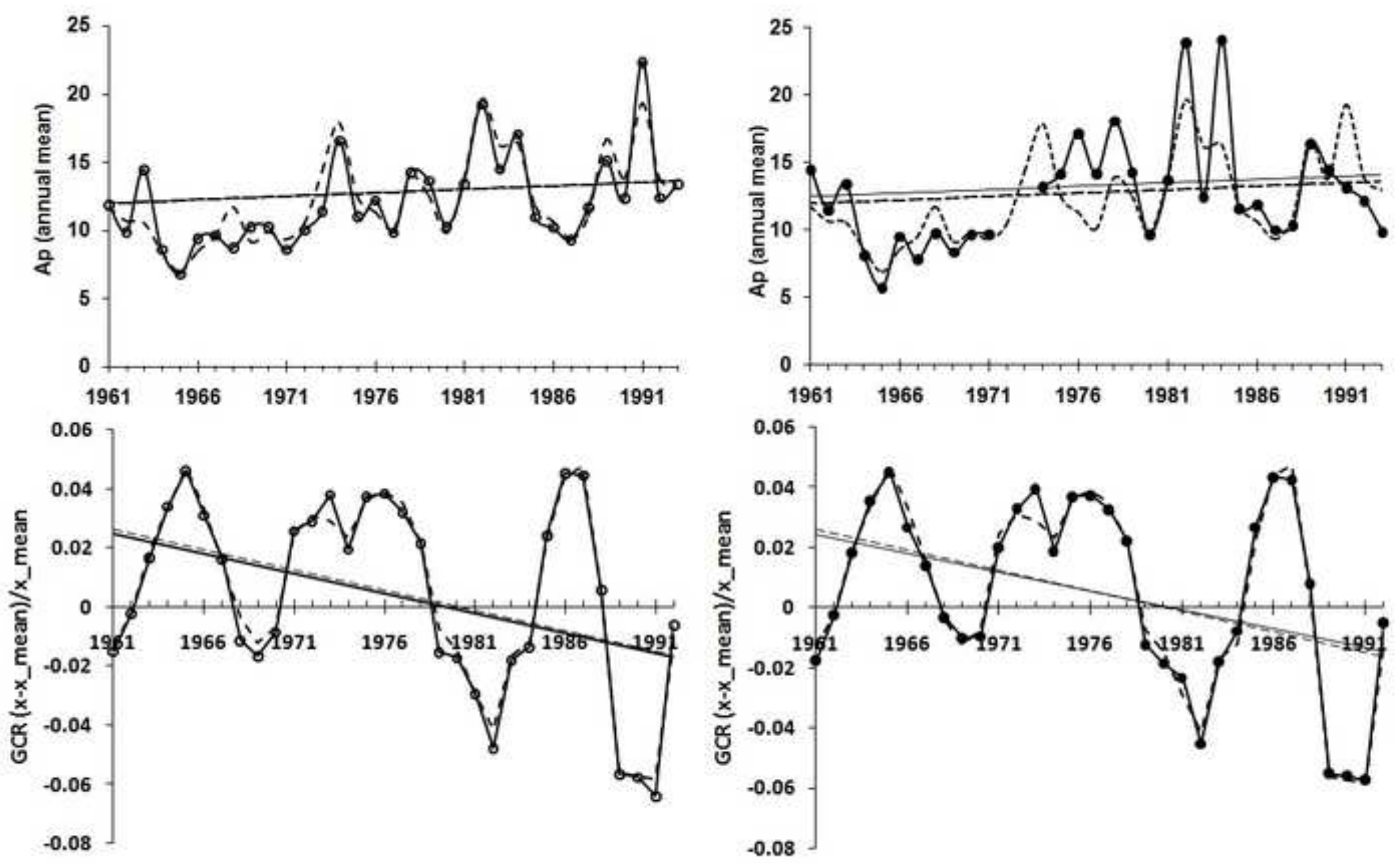